\title{
The Effect of Solvents, Acetone, Water, and Ethanol, on the Morphological and Optical Properties of ZnO Nanoparticles Prepared by Microwave
}

\author{
Phindile B. Khoza, ${ }^{1}$ Makwena J. Moloto, ${ }^{2}$ and Lucky M. Sikhwivhilu ${ }^{3}$ \\ ${ }^{1}$ Department of Chemical Technology, University of Johannesburg, P.O. Box 17011, Doornfontein 2028, South Africa \\ ${ }^{2}$ Department of Chemistry, Vaal University of Technology, Private Bag X021, Vanderbijlpark 1900, South Africa \\ ${ }^{3}$ Nanotechnology Innovation Centre, Advanced Materials Division, Mintek, Private Bag X3015, Randburg, \\ Johannesburg 2125, South Africa
}

Correspondence should be addressed to Makwena J. Moloto, makwenam@vut.ac.za

Received 16 September 2011; Revised 25 November 2011; Accepted 30 November 2011

Academic Editor: Chuan Jian Zhong

Copyright () 2012 Phindile B. Khoza et al. This is an open access article distributed under the Creative Commons Attribution License, which permits unrestricted use, distribution, and reproduction in any medium, provided the original work is properly cited.

\begin{abstract}
HDA-capped $\mathrm{ZnO}$ nanoparticles were prepared by solvothermal method using solvents of different polarities. A number of parameters were kept constant such as temperature, pressure, time, and $\mathrm{pH}$ while solvents were varied, that is, water, ethanol, and acetone. The TEM was used for the structural properties and morphologies such as spheres, mixture of rods, and spheres and stars were obtained in ethanol, acetone, and water, respectively, in a given reaction time of 15 minutes. Both ethanol and acetone gave rods with high aspect ratio primarily because of the lengths of the rods. Water and ethanol have the hydroxyl groups which interact with nanoparticles from nucleation, growth, and termination giving rise to nonspherical shapes. The hydroxyl group promotes growth in a nonuniform way resulting in stars and rods. The optical features were typical of $\mathrm{ZnO}$ nanoparticles with excitonic peaks in the range 368 to $374 \mathrm{~nm}$ from their absorption spectra. The XRD patterns of the particles gave the most stable form of $\mathrm{ZnO}$ which is the hexagonal phase, with high degree of crystallinity and with the 101 plane predominant in all solvents.
\end{abstract}

\section{Introduction}

Semiconductors in a nanoscale level have attracted a great deal of intrest due to their unique properties [1]. One of the unique characteristic of the nanoparticles is their size dependent electrical and optical properties known as the quantum confinement effect $[2,3]$. $\mathrm{ZnO}$ is one of the few semiconductors which exhibit quantum confinement effect. $\mathrm{ZnO}$ is a wide band gap $(3.34 \mathrm{eV})$ material and it has found applications in various fields [4]. It is an environmental friendly oxide, because of its nontoxicity and the ability to absorb in the UV range. It is as such used as a UV absorbent in the sunscreens and in solar energy conversion [5].

Other applications of $\mathrm{ZnO}$ include photovoltaic devices, gas sensors, photocatalysis, transparent conducting coating, and electrostatic transducers, [4]. It is well documented that the shape and size of the material strongly affect the properties and the applications of the material [6-9]. Hence, much effort is being dedicated on controlling the size and shape of the particles; however this still remains a challenge in synthetic chemists in the nanoscience field. Different factors such as time, temperature, concentration, precursors, capping molecule, solvents, and others are reported to affect the shape and size of the nanoparticles $[7,8]$.

$\mathrm{ZnO}$ is an interesting metal oxide because it can exist in diverse convoluted morphologies $[10,11]$. Herein we report on the effect of solvent effect on the shape of $\mathrm{ZnO}$ nanoparticles. Solvents play a crucial role in a reaction; they provide a means of temperature control by determining the highest temperature at which the reaction will occur. $\mathrm{ZnO}$ has been prepared by different methods. A novel solgel technique for the synthesis of stable zinc oxide colloids composed of nano-sized wurzite crystal was reported by Bahnemann et al. [12] and Spanhel and Anderson [13]. 
Other methods for the preparation of the metal oxide semiconductor nanoparticles reported to date include double-jet precipitation [14], flow through supercritical water method (FT-SCW) [15], and reverse micelles [16]. A nonhydrolytic single source precursor for the synthesis of the different metal oxide was reported by Rochengerger et al. [17] where a metal cupferron complex acts as a single source precursor for the various metal oxides. In a solvothermal synthesis, a solvent acts as a reaction medium that allows the relatively high temperature required for crystallisation of inorganic material [18]. It has proven to be the most efficient and easiest method for the production of $\mathrm{ZnO}$ nanoparticles. The choice of solvent used for the reaction has been reported to affect the shape of $\mathrm{ZnO}$ nanoparticles formed. Tonto and coworkers showed that by using organic solvents of different chain lengths, single crystalline $\mathrm{ZnO}$ nanoparticles of different aspect ratio can be prepared [18].

Another recent work on solvothermal synthesis is that reported by Linping $\mathrm{Xu}$ and coworkers. They reported that by using solvents of different saturated vapour pressures morphologies such as cauliflower, truncated, hexagonal, conical, spheres, rods, tubular, hour glass $\mathrm{ZnOs}$ are attained in solvents such as THF, decane, acetone, ethanol, water, and toluene, respectively [19]. However, the shapes were attained using conventional heating while microwave heating is also gaining importance in synthetic chemistry due to its unique effects such as rapid and selective heating, high reaction rate, increased product yield, and energy saving, and it has been used in the synthesis of $\mathrm{ZnO}$ nanoparticles [20-22]. Previously a mixture of hexadecylamine and oleic acid through hot reduction of zinc halide by superhydride followed by oxidation was reported to produce $\mathrm{ZnO}$ nanocrystals with low-size dispersion [23]. Herein we report on the effect of solvents polarity on the morphology and optical properties of $\mathrm{ZnO}$ nanoparticles produced by microwave irradiation.

\section{Experimental}

2.1. Materials. Zinc nitrate hexahydrate $\left(\mathrm{ZnNO}_{3} \cdot 6 \mathrm{H}_{2} \mathrm{O}\right)$ $99 \%$, sodium hydroxide pellets $(\mathrm{NaOH})$, hexadecylamine (HDA), ethanol, and acetone were reagents from SigmaAldrich and were all used without further purification.

2.2. Synthesis of Zinc Oxide Nanoparticles. Zinc oxide nanoparticles of various morphologies were synthesized using the method reported by Bahnemann et al. [12]. In a typical synthesis, $2.5 \mathrm{~g}$ of HDA, zinc nitrate hexahydrate $(0.3 \mathrm{M})$, and sodium hydroxide $(0.3 \mathrm{M})$ prepared by dissolving in either acetone, ethanol, or deionised water was placed into the teflon reaction vessels of the Multiwave 300 . The mixture was reacted at $120^{\circ} \mathrm{C}$ for $15 \mathrm{~min}$ in all solvent medium.

\subsection{Characterisation}

2.3.1. Optical Properties. Absorption spectra were acquired on a Analytikjena Specord 50 UV-Vis spectrophotometer. The particles were dissolved in methanol, and solution was placed in a quarts cuvettes with $1 \mathrm{~cm}$ path length. A Perkin
Elmer LS 45 Fluorimeter was used for the photoluminescence of the nanoparticles dissolved in methanol.

2.3.2. Electron Microscopy. Transmission electron microscopy was acquired on the Hitachi Jeol $100 \mathrm{~S}$ operated at $80 \mathrm{keV}$. A drop of nanoparticles dissolved in methanol was placed on a copper grid.

2.3.3. Powder X-Ray Diffraction. XRD patterns of the powdered samples were obtained on a Phillips X'Pert materials research diffractometer using secondary monochromated $\mathrm{Cu} \mathrm{K} \alpha$ radiation $(\lambda=1.54060 \AA)$ at $40 \mathrm{Kv} / 50 \mathrm{~mA}$. Samples were supported on a glass slide. Measurements were taken using a glancing angle of incidence detector at an angle of 2 for $2 \theta$ values over $10-80$ in steps of 0.05 with a scan speed of 0.012 .

2.3.4. Fourier Transform Infrared (FTIR) Spectroscopy. The FT-IR spectrum of the nanoparticles was recorded on a Perkin Elmer Spectrum 100 FTIR spectrometer. The powder sample was placed on a sample holder and the spectrum was recorded.

\section{Results and Discussions}

The synthesis of $\mathrm{ZnO}$ nanoparticles was made in various methods to study the effects on conditions such as precursors, temperature, time, and concentration on the morphology and properties of nanoparticles. The solvents have not been investigated to explore their effects in the morphologies and optical properties. In the work various solvents with varying degree of polarities were used. One of the general morphologies obtained from aqueous media is star-shaped nanoparticles, and different morphologies were obtained in solvents acetone, water, and ethanol due to the solvent interactions with the precursor, zinc nitrate salt in the presence of hexadecylamine to stabilize the particles. Microwave technique was used to ensure that the precursor is soluble in all solvents and hexadecylamine also was dispersed to allow both kinetic and thermodynamic control in the growth of nanoparticles. The interaction between solvents, hexadecylamine, and the precursor becomes critical in influencing the growth of particles. The addition of a base sodium hydroxide is generally known to generate an intermediate hydroxide $\mathrm{Zn}(\mathrm{OH})_{4}{ }^{2-}$ upon reaction with the zinc salt (1). The conversion of the hydroxides into $\mathrm{ZnO}$ (2) is promoted by excess of sodium hydroxide in the presence of solvent medium either water, ethanol, or acetone. Water has the least solubility towards HDA whereas in acetone and ethanol solubility is increased and allowing more interaction between the particles and the capping molecule:

$$
\begin{gathered}
\mathrm{Zn}^{2+}+4 \mathrm{OH}^{-} \longrightarrow \mathrm{Zn}(\mathrm{OH})_{4}{ }^{2-} \\
\mathrm{Zn}(\mathrm{OH})_{4}{ }^{2-} \longrightarrow \mathrm{ZnO}
\end{gathered}
$$




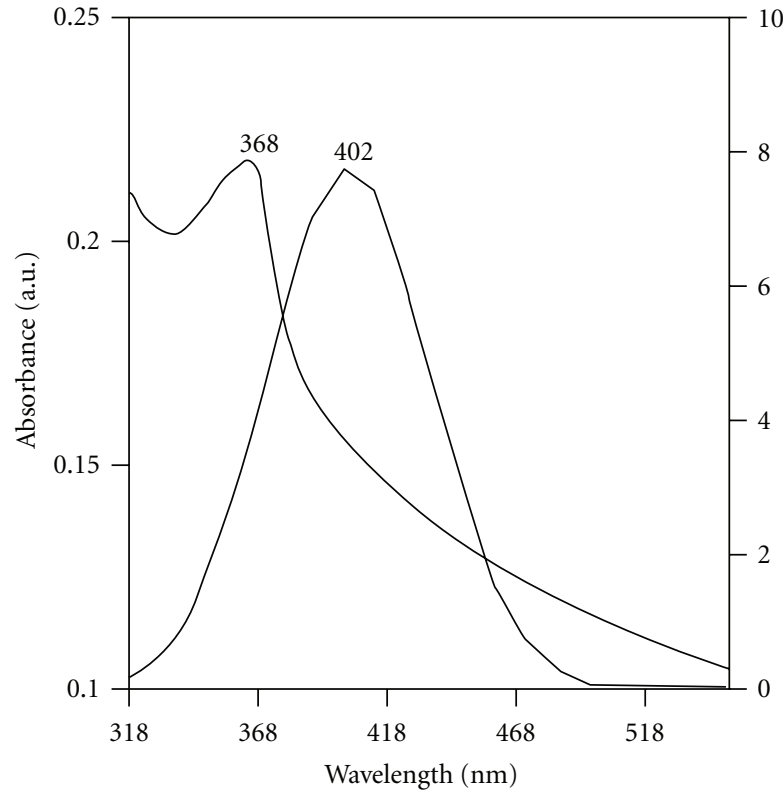

(a)
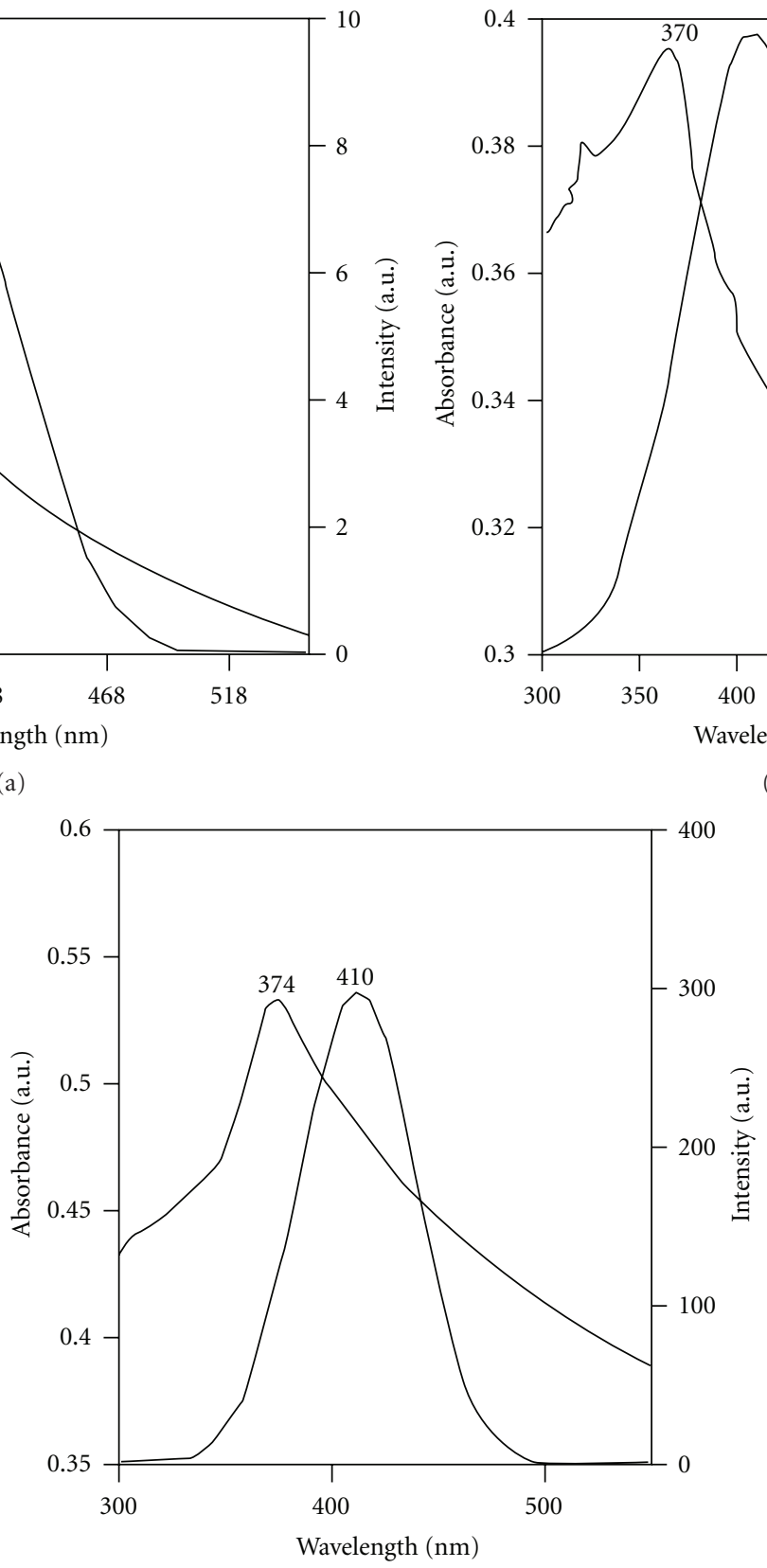

(c)

FIGURE 1: Absorption and emission spectra of $\mathrm{ZnO}$ nanoparticles synthesised in ethanol (a), acetone (b), and water (c) at $120^{\circ} \mathrm{C}$ and time of 15 minutes under microwave.

3.1. Optical Properties. The optical properties of $\mathrm{ZnO}$ nanoparticles in ethanol as a solvent are shown in Figure 1(a). $\mathrm{ZnO}$ normally exhibits two emission peaks; one is a broad green emission in the region between 500 and $530 \mathrm{~nm}$ [10] which is due to the oxygen vacancy. The other gave much narrow ultraviolet emission band at around $400 \mathrm{~nm}$ which is due to the excitons recombination. A welldefined excitonic peak appears at $368 \mathrm{~nm}$ and is slightly blue shifted when compared to the bulk which appears at $370 \mathrm{~nm}$. One of the impressive features of semiconductor nanoparticles is their ability to emit light upon excitation

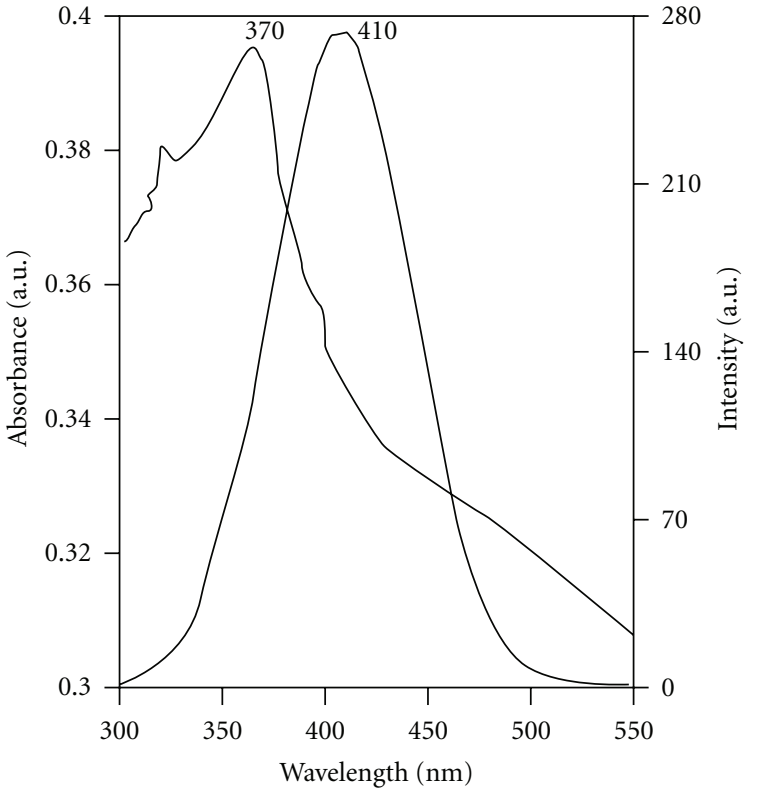

(b) 


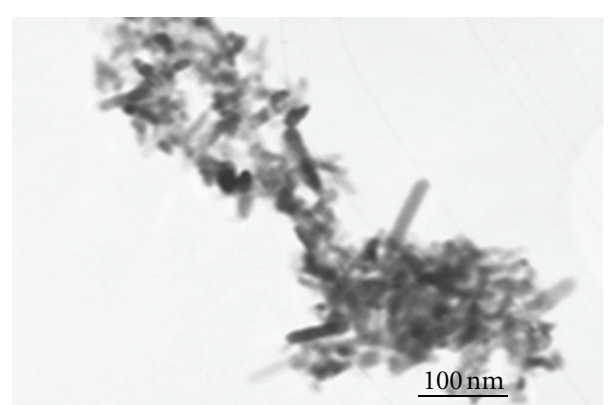

(a)

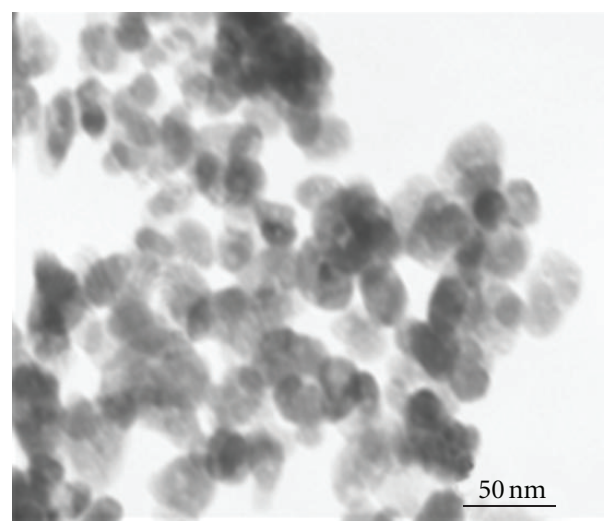

(c)

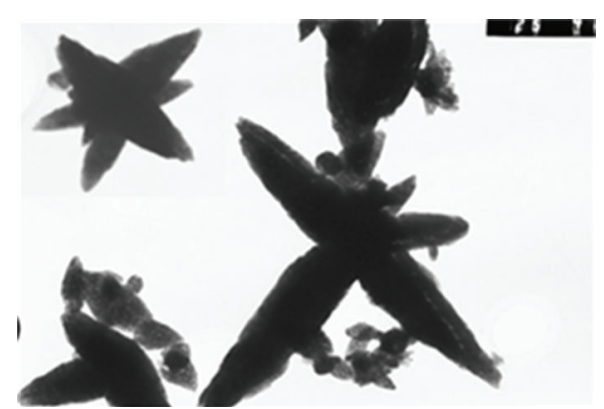

(b)

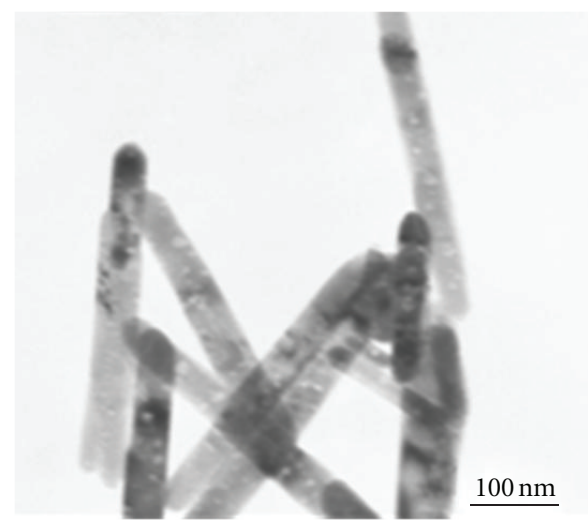

(d)

FIGURE 2: TEM images of $\mathrm{ZnO}$ nanoparticles synthesised in various solvents, ethanol (a), acetone (b, c), and water (d) at $120^{\circ} \mathrm{C}$ and time of 15 minutes under microwave.

is due to the particles nanosize regime exhibiting quantum confinement effect. The photoluminescence spectra show a broad emission due to the wide distribution of the particle size. The large particle size and shape of the particles synthesised in water as a solvent influenced the appearance of the absorption peak (Figure 1(c)). The absorption peak for water is also much more tailing compared to the peak of acetone and ethanol.

\subsection{Structural Properties}

3.2.1. TEM and IR Spectral Analysis. In the alkyl containing solvents, ethanol, and acetone, a mixture of rods and spheres was formed. It has been reported that organic solvents promote the formation of spheres and rods [19]; therefore a similar trend was observed with ethanol and acetone (Figures 2(a), 2(b, c)), respectively. Particles formed when ethanol was used as a solvent in Figure 2(a) have spheres ranging from 20 to $60 \mathrm{~nm}$ and rods with aspect ratio of 8-60. Since nanoparticles are so small, they are very unstable and tend to agglomerate. Over the years the problem has been overcome by using an organic molecule that is going to introduce steric hindrance between the particles thereby preventing agglomeration. Hexadecylamine (HDA) has proven to be a good capping agent over the years. It is versatile and is mobile enough to readily add to monomer but stable enough to prevent agglomeration [6]. Even though HDA is such a good capping agent, particles obtained in ethanol appear to have agglomerated. It is well documented that $\mathrm{ZnO}$ contains the fastest growing plan 0001. Therefore in the beginning of the reaction, spheres are formed which contains the high energy 0001. Because of the high energy, the plane that elongates faster than the other planes resulting into the formation of rods [24].

Particles formed in acetone (Figures 2(b) and 2(c)) have spheres with sizes ranging from 45 to $100 \mathrm{~nm}$ and rods with aspect ratio which ranges from 37 to 94 , and particles are bigger and longer than those formed in ethanol. Acetone has the lowest boiling point compared to ethanol; therefore particles formed in acetone are heated to a lower temperature, and because of the low dielectric constant compared to ethanol, it interacts the least with the electromagnetic radiation of the microwave. Therefore particles formed in acetone were expected to be much smaller. Long rods also formed in acetone a phenomenon established when nonalcoholic solvents are sued in solvothermal synthesis [25] of $\mathrm{ZnO}$ nanoparticles. The influence in the formation of long rods could be resulting from the one-dimensionality of the interaction with the amine group of the HDA. HDA is known to promote growth along one plane through the bonding interaction between the $\mathrm{NH}_{2}$ group and the $\mathrm{ZnO}$ nanoparticles. Therefore the solubility of HDA in acetone at the given temperature $\left(120^{\circ} \mathrm{C}\right)$ allows the formation of both spheres and rods under the microwave treatment. Using water as solvent, star-shaped particles were formed (Figure 2(d)). The star-shaped nanoparticles have also been 


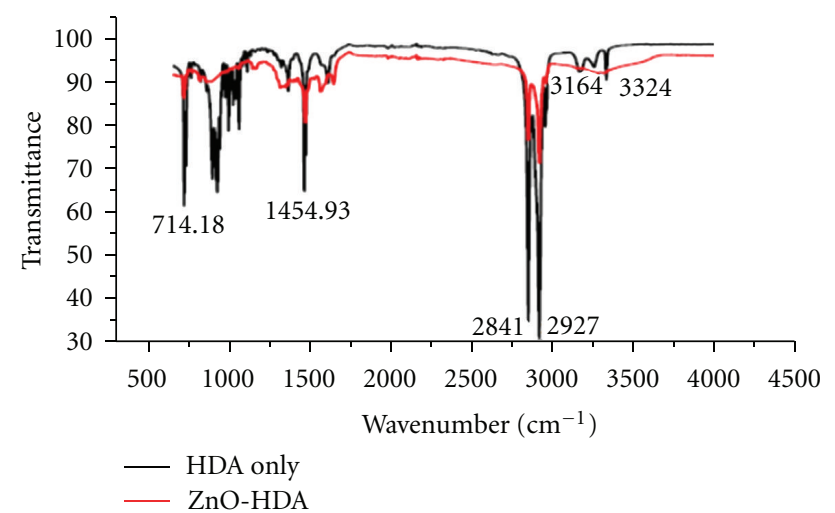

FIGURE 3: FTIR spectra of $\mathrm{ZnO}$ nanoparticles synthesised using zinc nitrate in water at $120^{\circ} \mathrm{C}$ and time of 15 minutes under microwave.

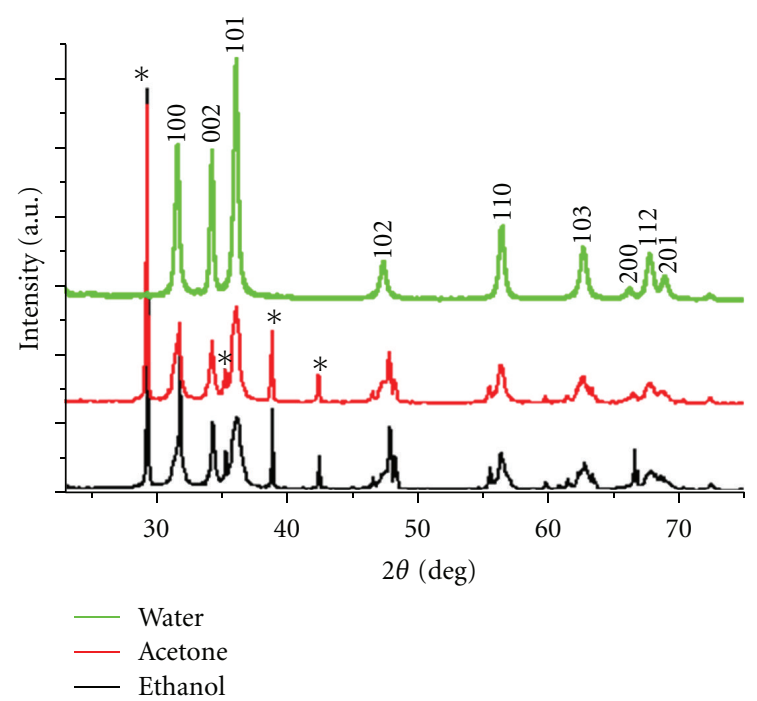

FIGURE 4: XRD pattern $\mathrm{ZnO}$ nanoparticles synthesised using zinc nitrate in different solvents, water, acetone, and ethanol at $120^{\circ} \mathrm{C}$ and time of 15 minutes under microwave.

reported using conventional heating; their shape which is due to the coalescence of several nuclei and water has the tendency of forming star-shaped particles [24].

Under normal conditions, HDA will not dissolve in water; however the microwave might induce the dissolution of HDA in water therefore capping the particles effectively. To confirm that HDA passivated the particles formed in water, the IR was used. The FTIR in Figure 3 illuminates the evolution of surface-bonded molecule on $\mathrm{ZnO}$ nanoparticles. In HDA only, the peaks at $3181.87-3336 \mathrm{~cm}^{-1}$ are due to $\mathrm{N}-\mathrm{H}$ stretching of the primary amine. The $\mathrm{C}-\mathrm{H}$ and C-N-H stretching appears at 2850 and $2916 \mathrm{~cm}^{-1}$. In $\mathrm{ZnO}$ capped with HDA, the $\mathrm{N}-\mathrm{H}$ vibration disappears and the peak broadens; this can be possible due to the $\mathrm{OH}$ stretching of some water on the surface of $\mathrm{ZnO}$ nanoparticles. The $\mathrm{C}-\mathrm{C}\left(714.8 \mathrm{~cm}^{-1}\right)$ and $\mathrm{CH}_{2}$ wagging $\left(1454.3 \mathrm{~cm}^{-1}\right)$ vibrations are highly intense in HDA due to the high number of $\mathrm{CH}_{2}$ 's in the molecular structure of HDA; however the introduction of $\mathrm{ZnO}$ nanoparticles weakens the peaks. The presence of similar peaks from both $\mathrm{ZnO}-\mathrm{HDA}$ and HDA cannot conclusively denote the interaction of HDA with $\mathrm{ZnO}$ nanoparticles but it does indicate the presence of HDA in the sample.

3.2.2. XRD Analysis. To confirm the purity and determine the phase of the particles, $X$-ray diffractometry was used. The $\mathrm{XRD}$ diffraction peaks were indexed to hexagonal phase $\mathrm{ZnO}$. The morphologies from acetone and ethanol (Figure 4) have similar diffraction patterns except for the relative intensities due to their random orientation. XRD pattern for water was indexed to pure hexagonal phase $\mathrm{ZnO}$ without any trace of Zinc Nitrate, while ethanol and acetone show some impurities marked with an asterisk. The impurities are from unreacted zinc nitrate; however the peaks are broad in both solvents compared to water which has narrow and more intense peaks. The broadness of the peaks is due to the small size of the particles.

\section{Conclusions}

The synthesis of $\mathrm{ZnO}$ nanoparticles capped with hexadecylamine (HDA) was carried out with $\mathrm{Zn}\left(\mathrm{NO}_{3}\right)_{2}$ and $\mathrm{NaOH}$ as the base in acetone, ethanol, and water. Reaction in ethanol results in the formation of rods and spheres which are of smaller sizes with respect to those formed in acetone as a solvent under similar conditions. Water as a solvent forms highly crystalline $\mathrm{ZnO}$ nanoparticles which are star shaped. The solvent influence was observed to the drive for the interaction of HDA with $\mathrm{ZnO}$ nanoparticles growth with both thermodynamic and kinetic controls considered under the microwave irradiation. The polar characteristic of the solvent was proposed to be the main factor that affects both nucleation and growth of $\mathrm{ZnO}$ nanoparticles and, consequently, determines the shape, size, and aspect ratio of the products.

\section{Acknowledgments}

The work was performed in the laboratories of the CSIRNCNSM in Pretoria, University of Johannesburg, Department of Chemical Technology, and Vaal University of Technology. The financial support was obtained from the National Research Foundation (NRF), South Africa. The electron microscope images were measured from the electron microscope unit at the University of Witwatersrand.

\section{References}

[1] M. A. El-Sayed, "Small is different: shape, size, and composition-dependent properties of some colloidal semiconductor nanocrystals," Accounts of Chemical Research, vol. 37, no. 5, pp. 326-333, 2004.

[2] N. L. Pickett and P. O'Brien, "Syntheses of semiconductor nanoparticles using single-molecular precursors," Chemical Records, vol. 1, no. 6, pp. 467-479, 2001.

[3] C. F. Landes, S. Link, M. B. Mohamed, B. Nikoobakht, and M. A. El-Sayed, "Some properties of spherical and rod-shaped 
semiconductor and metal nanocrystals," Pure and Applied Chemistry, vol. 74, no. 9, pp. 1675-1692, 2002.

[4] Y. S. Fu, X. W. Du, S. A. Kulinich et al., "Stable aqueous dispersion of $\mathrm{ZnO}$ quantum dots with strong blue emission via simple solution route," Journal of the American Chemical Society, vol. 129, no. 51, pp. 16029-16033, 2007.

[5] A. Becheri, M. Dürr, P. Lo Nostro, and P. Baglioni, "Synthesis and characterization of zinc oxide nanoparticles: application to textiles as UV-absorbers," Journal of Nanoparticle Research, vol. 10, no. 4, pp. 679-689, 2008.

[6] N. Moloto, M. J. Moloto, N. J. Coville, and S. Sinha Ray, "Optical and structural characterization of nickel selenide nanoparticles synthesized by simple methods," Journal of Crystal Growth, vol. 311, no. 15, pp. 3924-3932, 2009.

[7] Y. W. Jun, J. S. Choi, and J. Cheon, "Shape control of semiconductor and metal oxide nanocrystals through nonhydrolytic colloidal routes," Angewandte Chemie-International Edition, vol. 45, no. 21, pp. 3414-3439, 2006.

[8] C. Tang, S. Fan, M. L. De La Chapelle, H. Dang, and P. Li, "Synthesis of gallium phosphide nanorods," Advanced Materials, vol. 12, no. 18, pp. 1346-1348, 2000.

[9] J. Yang, J. H. Zeng, S. H. Yu, L. Yang, G. E. Zhou, and Y. T. Qian, "Formation process of CdS nanorods via solvothermal route," Chemistry of Materials, vol. 12, no. 11, pp. 3259-3263, 2000.

[10] D. Sun, H. J. Sue, and N. Miyatake, "Optical properties of ZnO quantum dots in epoxy with controlled dispersion," Journal of Physical Chemistry C, vol. 112, no. 41, pp. 16002-16010, 2008.

[11] Z. L. Wang, "Zinc oxide nanostructures: growth, properties and applications," Journal of Physics Condensed Matter, vol. 16, no. 25, pp. R829-R858, 2004.

[12] D. W. Bahnemann, C. Kormann, and M. R. Hoffmann, "Preparation and characterization of quantum size zinc oxide: a detailed spectroscopic study," Journal of Physical Chemistry, vol. 91, no. 14, pp. 3789-3798, 1987.

[13] L. Spanhel and M. A. Anderson, "Semiconductor clusters in the sol-gel process: quantized aggregation, gelation, and crystal growth in concentrated $\mathrm{ZnO}$ colloids," Journal of the American Chemical Society, vol. 113, no. 8, pp. 2826-2833, 1991.

[14] Q. Zhong and E. Matijević, "Preparation of uniform zinc oxide colloids by controlled double-jet precipitation," Journal of Materials Chemistry, vol. 6, no. 3, pp. 443-447, 1996.

[15] K. Sue, M. Suzuki, K. Arai et al., "Size-controlled synthesis of metal oxide nanoparticles with a flow-through supercritical water method," Green Chemistry, vol. 8, no. 7, pp. 634-638, 2006.

[16] A. K. Gangul, S. Vaidya, and T. Ahmad, "Synthesis of nanocrystalline materials through reverse micelles: a versatile methodology for synthesis of complex metal oxides," Bulletin of Materials Science, vol. 31, no. 3, pp. 415-419, 2008.

[17] J. Rockenberger, E. C. Scher, and A. P. Alivisatos, "A new nonhydrolytic single-precursor approach to surfactantcapped nanocrystals of transition metal oxides," Journal of the American Chemical Society, vol. 121, no. 49, pp. 11595-11596, 1999.

[18] P. Tonto, O. Mekasuwandumrong, S. Phatanasri, V. Pavarajarn, and P. Praserthdam, "Preparation of $\mathrm{ZnO}$ nanorod by solvothermal reaction of zinc acetate in various alcohols," Ceramics International, vol. 34, no. 1, pp. 57-62, 2008.

[19] L. Xu, Y. L. Hu, C. Pelligra et al., "ZnO with different morphologies synthesized by solvothermal methods for enhanced photocatalytic activity," Chemistry of Materials, vol. 21, no. 13, pp. 2875-2885, 2009.
[20] S. Komarneni, M. Bruno, and E. Mariani, "Synthesis of $\mathrm{ZnO}$ with and without microwaves," Materials Research Bulletin, vol. 35, no. 11, pp. 1843-1847, 2000.

[21] S. A. Galema, "Microwave chemistry," Chemical Society Review, vol. 26, p. 233, 1997.

[22] K. J. Rao, B. Vaidhyanathan, M. Ganguli, and P. A. Ramakrishnan, "Synthesis of inorganic solids using microwaves," Chemistry of Materials, vol. 11, no. 4, pp. 882-895, 1999.

[23] P. D. Cozzoli, A. Kornowski, and H. Weller, "Colloidal synthesis of organic-capped $\mathrm{ZnO}$ nanocrystals via a sequential reduction-oxidation reaction," Journal of Physical Chemistry $B$, vol. 109, no. 7, pp. 2638-2644, 2005.

[24] S. Cho, S. H. Jung, and K. H. Lee, "Morphology-controlled growth of $\mathrm{ZnO}$ nanostructures using microwave irradiation: from basic to complex structures," Journal of Physical Chemistry C, vol. 112, no. 33, pp. 12769-12776, 2008.

[25] S. K. N. Ayudhya, P. Tonto, O. Mekasuwandumrong, V. Pavarajarn, and P. Praserthdam, "Solvothermal synthesis of $\mathrm{ZnO}$ with various aspect ratios using organic solvents," Crystal Growth and Design, vol. 6, no. 11, pp. 2446-2450, 2006. 

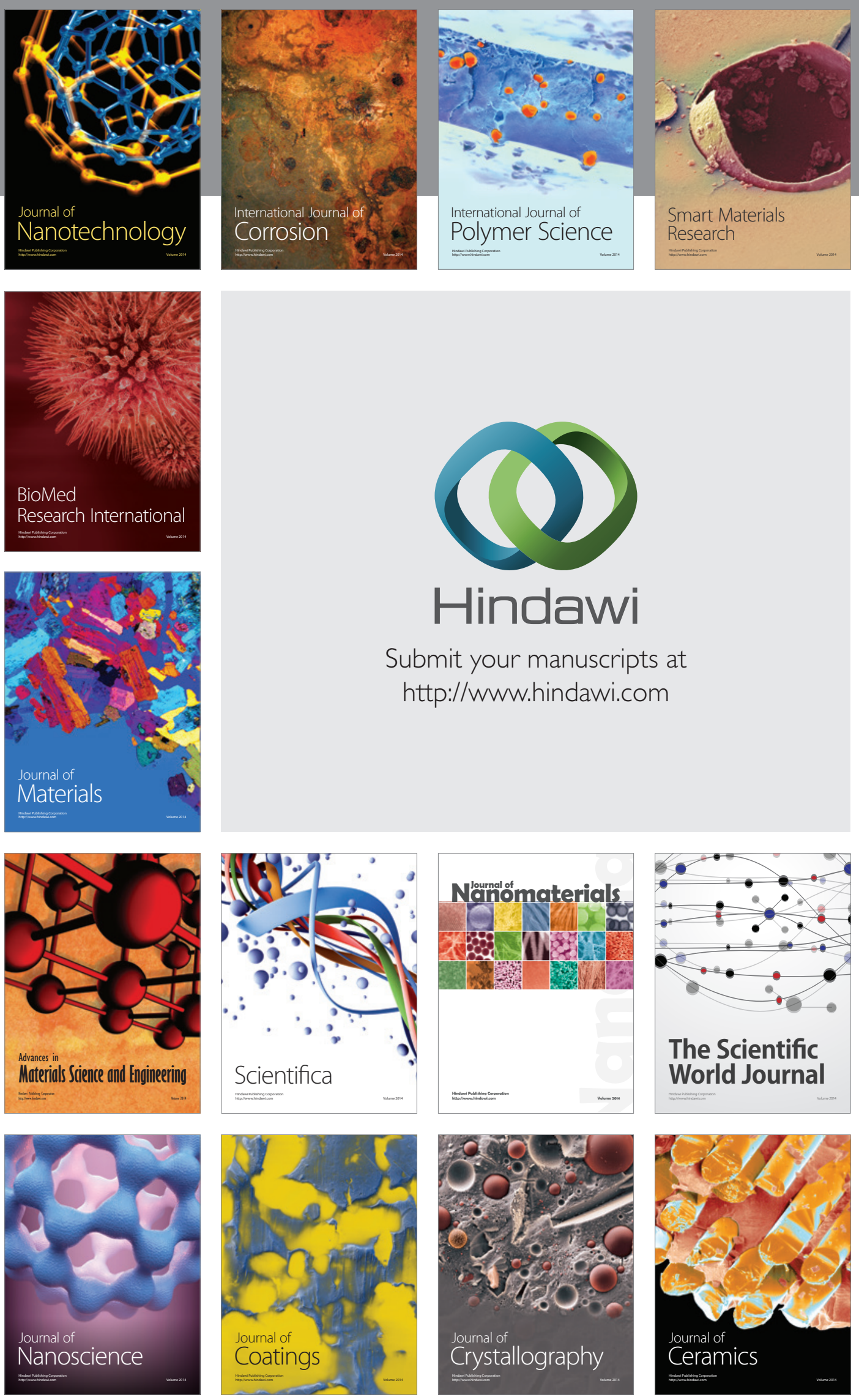

The Scientific World Journal

Submit your manuscripts at

http://www.hindawi.com

\section{World Journal}

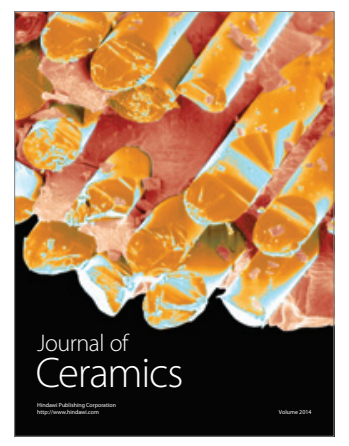

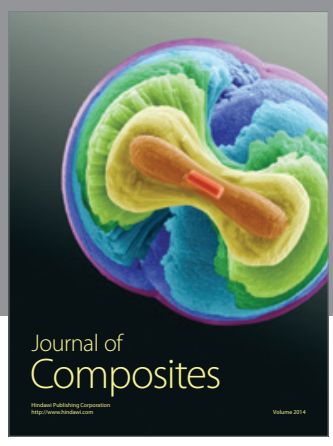
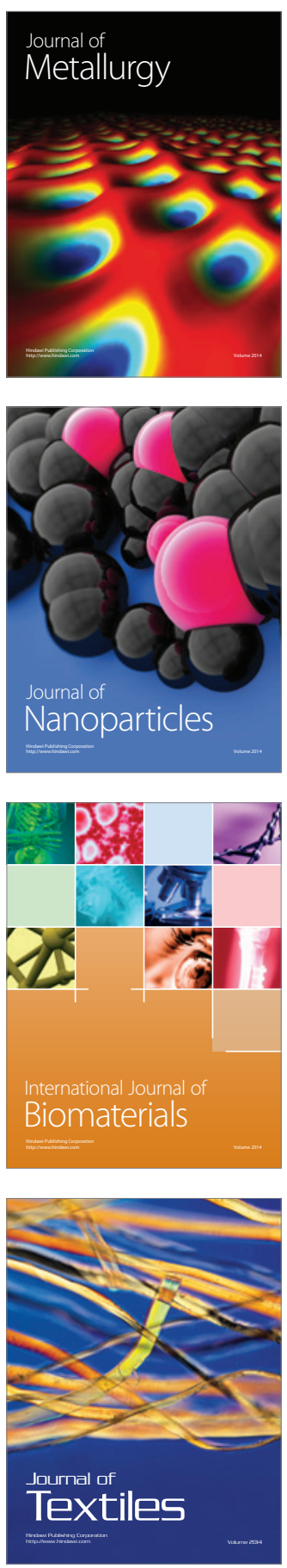